Classes I and II than in III-V. Survival of kernicteric babies and dyskinetic cases was higher, due to better infant care in Social Classes I and II than in lower social classes.

\title{
TREATABLE GLUT 1 DEFICIENCY CHOREA SYNDROME
}

A 7-year-old developmentally delayed girl with chorea and a glucose transporter type 1 (GLUT 1) deficiency syndrome is reported from Hospital Universitari Sant Joan de Deu, Barcelona, Spain, and University of Texas Southwestern Medical Centre, Dallas. At 5 years of age she presented with an abrupt flaccidity and loss of ambulation for several minutes, without loss of consciousness. Chorea and mental retardation were associated with paroxysmal ataxia, convergent strabismus, dysarthria, dystonia, and aggravated intellectual disability induced by fasting or exertion. MRI showed cerebral hypotrophy, especially occipital lobes, and enlarged ventricles. EEG showed mild diffuse slowing, maximal posteriorly. Blond glucose and lactate were normal, but CSF glucose and lactate were diminished. DNA sequencing revealed a sporadic, heterogeneous amino acid insertion in the GLUT 1 transporter that probably impaired blood-brain glucose flux. Treatment with the ketogenic diet was followed by brain growth and improved symptomatic outcome by 7 years of age. Intellectual deterioration was unaffected, remaining below the $5^{\text {th }}$ percentile for age. (Perez-Duenas B, Prior C, Ma Q, et al. Childhood chorea with cerebral hypotrophy. A treatable GLUT 1 energy failure syndrome. Arch Neurol Nov 2009;66(11):1410-1414). (Respond: Juan M Pascual MD $\mathrm{PhD}$, University of Texas Southwestern Medical Center, 5323 Harry Hines Boulevard, Mail Code 8813, Dallas, TX 75390. E-mail: Juan.Pascual@UTSouthwestern.edu).

COMMENT. Most patients with GLUT 1 deficiency syndrome present with epilepsy and deceleration of brain growth, and the above case is considered a novel phenotype. Whereas chorea and ataxia were controlled by treatment with the ketogenic diet, intellectual deterioration was not reversed.

\section{TICS, OCD AND ADHD ASSOCIATED WITH BRAIN TUMOR}

Researchers at Children's Hospital of Michigan, Detroit report a boy aged 12 years with a 12-month history of ADHD, OCD, and stimulant-induced tourette syndrome that resolved following surgical removal of a right temporal lobe and basal ganglia oligodendroglioma. He had also developed complex partial seizures and two episodes of secondary generalized tonic clonic seizures. Oxcarbazepine controlled the seizures, and atomoxetine for ADHD was gradually withdrawn after surgery with no relapse of behavior. Radiation therapy was used for the residual tumor that increased in size 2 years after lesionectomy. Neuropsychological testing 3.5 years after surgery indicated normal verbal intellectual, memory, receptive language, and achievement, with impaired nonverbal, naming, and manual dexterity. Attentional, OCD, and anxiety symptoms resolved postoperatively. (Luat AF, Behen ME, Juhasz C, Sood S, Chugani HT. Secondary tics or tourettism associated with a brain tumor. Pediatr Neurol Dec 2009;41:457-460). (Dr Chugani, Pediatric Neurology/PET Center, Children's Hospital of Michigan, 3901 Beaubien Blvd, Detroit, MI 48201. E-mail: hchugani@ pet.wayne.edu). 
COMMENT. The authors recommend that children who present with ADHD, OCD, or tic disorder of late onset and in the absence of a family history should be investigated with brain MRI. This report emphasizes the importance of a neurological basis for ADHD and tics, and the need to exclude structural lesions in the cerebrum and connections with the basal ganglia. Children with ADHD of a genetic origin alone develop symptoms early, before 7 years of age. Other structural lesions sometimes associated with ADHD include temporal lobe arachnoid cyst (Millichap JG, Neurology 1997;48:1435-1439), decreased size of splenium of corpus callosum (Semrud-Clikeman $\mathrm{M}$ et al, 1994), and decreased volume of prefrontal cortex, caudate nucleus, and globus pallidum (Castellanos FX et al, 1996).

Two children who presented with chorea, athetosis and dystonia were diagnosed with astrocytoma of the thalamus and a pontine midbrain tumor. (Millichap JG, Miller RH, Backus RE, JAMA 1962;179:589-593). Diagnoses of rheumatic chorea, dystonia musculorum deformans, and encephalitis were, entertained before fractional pneumoencephalography defined an expanding lesion. Of 300 intracranial tumors in children treated at the Mayo Clinic between 1950 and 1960, 4 per cent involved the basal ganglia, but less than 1 per cent were associated with involuntary movements. A review of the literature found only 9 similar cases between 1923 and 1961.

\section{ATTENTION DEFICIT AND LEARNING DISORDERS}

\section{TOBACCO AND LEAD EXPOSURES AND ADHD}

The independent and joint effects of prenatal tobacco and childhood lead exposures on ADHD in a national sample of US children were studied by researchers at Cincinnatti Children's Hospital, OH; British Columbia Children's Hospital, Vancouver, Canada; and University of North Carolina, Chapel Hill, NC. Using data from a 20012004 National Health and Nutrition Examination Survey, a total of $8.7 \%$ of children 8 15 years of age met criteria for ADHD. Prenatal tobacco exposure and third tertile (1.3-5 $\mathrm{mcg} / \mathrm{dL}$ ) current blood lead concentrations were independently associated with ADHD. (Children with lead levels $>5 \mathrm{mcg} / \mathrm{dL}$ were excluded). Compared with children with neither exposure, children with both exposures had an even greater risk of ADHD than if the independent risks were multiplied $(\mathrm{p}<0.001)$. Children who attended preschool and boys (vs girls) had increased likelihoods of ADHD. Mexican American and black children had lower risks compared to non-Hispanic white children. (Froehlich TE, Lanphear BP, Auinger P, et al. Association of tobacco and lead exposures with attentiondeficit hyperactivity disorder. Pediatrics Dec 2009;124:e1054-e1063). (Respond: Tanya Froehlich MD, Cincinnati Children's Hospital Medical Center, 3333 Burnet Ave, Mail Location 4002, Cincinnati, OH 45229. E-mail: tanya.froelich@ cchmc.org).

COMMENT. The association of prenatal tobacco exposure and ADHD has been demonstrated in previous studies, one involving a national data base population, but this is the first indication of a potentiation of effect when combined with childhood low level lead exposure. A similar association with ADHD is reported with a combination tobacco and alcohol prenatal exposure. Patients with ADHD are 2.5 times more likely to be exposed to alcohol in utero and 2.1 times more likely to be exposed to tobacco. Alcohol 